\title{
Estimación de pérdidas económicas causadas por Trypanosoma vivax en un rodeo lechero de Argentina
}

\author{
Abdala, A.A. ; Larriestra, A.J.'; Signorini, M. ${ }^{3}$ \\ ${ }^{1}$ INTA-EEA, Rafaela, Santa Fe Argentina. ${ }^{2}$ Facult.Agron.y Vet.UNRC, Córdoba. \\ ${ }^{3}$ CONICET-INTA-EEA Rafaela, Santa Fe, Argentina. \\ E-mail: abdala.alejandro@inta.gob.ar
}

\begin{abstract}
Resumen
Abdala, A.A.; Larriestra, A.J.; Signorini, M.: Estimación de pérdidas económicas causadaspor Trypanosoma vivax en un rodeo lechero de Argentina. Rev. Vet. 31: 2, 115-119, 2020. En Argentina T. vivax fue descrito por primera vez en 2006 afectando ganado de carne de la Provincia de Formosa. Durante el verano/otoño de 2016/2017 se observaron los primeros brotes en la zona central de la Provincia de Santa Fe, afectando algunos rodeos lecheros. Se describe el impacto que este hemoparásito produjo en un establecimiento lechero con 220 vacas totales, donde los síntomas principales fueron abortos $(n=17)$, diarrea, mala condición corporal, baja o nula producción de leche y muerte de vacas $(7,5 \%)$. Las pérdidas totalizaron 58.802 USD considerando los abortos, muertes de vacas, venta de animales improductivos y gastos por tratamiento específico. Por tratarse de una enfermedad desconocida para la zona, la demora en realizar el diagnóstico exacerbó las pérdidas. El tratamiento de la totalidad de los bovinos adultos con diminazene detuvo el brote, siendo económicamente justificable su aplicación.
\end{abstract}

Palabras clave: ganado lechero, Trypanosoma vivax, pérdidas económicas, Argentina.

\begin{abstract}
Abdala, A.A.; Larriestra, A.J.; Signorini, M.: Estimate of economic losses caused by Trypanosoma vivax in a dairy rodeo of Argentina. Rev. Vet. 31: 2, 115-119, 2020. In Argentina, bovine trypanosomiasis is caused by Trypanosome vivax. Outbreaks of the disease were first described in beef cattle at the Formosa Province in 2006 and in dairy herds from the central area of Santa Fe Province, during the summer and autumn of 2017. Outbreaks were characterized by diarrhea, decay of body condition, severe drop of milk production, abortions and death. A herd with 220 dairy cows, in which the identification of the disease was belated, reported 17 abortions and $7.5 \%$ of deaths. The outbreak was temporarily stopped by treatment of the whole herd with diminazene. The economic impact of the outbreak, considering abortions, treatments, culling of cows and death, achieved US\$ 58,802. Early treatment of all cattle would be cost-effective given the severe economic impact caused by the disease when control measures are delayed.
\end{abstract}

Key words: dairy cattle, Trypanosoma vivax, economic losses, Argentina.

\section{INTRODUCCIÓN}

Tripanosoma vivax es un hemoparásito que afecta animales ungulados domésticos y silvestres en África, Centro y Sudamérica. El parásito es principalmente transmitido por la mosca tse-tse y otros insectos hematófagos de África. En América, la mosca brava (Stomoxis calcitrans) y los tábanos (Tabanidae sp) son los responsables de la transmisión mecánica entre animales infectados y sanos ${ }^{16}$. La enfermedad cursa con anemia y emaciación, pudiendo causar la muerte de los animales.

En África, las pérdidas económicas que ocasiona esta parasitosis solo por el uso de drogas específicas

Recibido: julio 2020 / Aceptado: septiembre 2020 para su tratamiento, fueron estimadas en el orden de los 35 millones de US\$ ${ }^{7}$. En base a pérdidas observadas en Brasil en siete rodeos de la región del Pantanal y extrapolando sus resultados a la totalidad de la población bovina en riesgo (11 millones de cabezas), se determinó que la pérdida potencial excedería los USD 160 millones ${ }^{19}$.

T. vivax fue reportado por primera vez en Argentina en el año 2006 en bovinos de carne de la provincia de Formosa ${ }^{14,15}$. Durante el verano-otoño de 2016 y 2017 se produjeron varios brotes en tambos de la zona central de la Provincia de Santa Fe. Los mismos se caracterizaron por producir abortos, diarreas, disminución de la producción y muerte de vacas. La introducción de este parásito en una zona indemne y de importante producción lechera, abre una serie de interrogantes sobre 
el impacto económico negativo que la actividad y los productores podrían sufrir.

El objetivo de este trabajo fue describir un brote de T. vivax en un rodeo lechero ubicado en el Departamento Castellanos (Provincia de Santa Fe, Argentina) y estimar sus pérdidas económicas y productivas asociadas.

\section{MATERIAL Y MÉTODOS}

Características del rodeo. El establecimiento lechero que sufrió el brote de tripanosomiasis está ubicado en el distrito de Ataliva $\left(30^{\circ} 59^{\prime} \mathrm{S}, 61^{\circ} 23^{\prime} \mathrm{O}\right)$ del Departamento Castellanos, Provincia de Santa Fe, contando con 220 vacas Holstein, sobre una superficie de 170 Ha. En este departamento y su aledaño Las Colonias, se concentran la mayor cantidad de tambos de Santa Fe. El clima es considerado subtropical húmedo, con una precipitación media anual entre 975 y $1200 \mathrm{~mm}$.

El establecimiento lechero desarrolla su actividad en base al pastoreo directo de pasturas cultivadas de alfalfa y gramíneas, con suplementación de concentrado y silo de maíz. El ordeño se realiza dos veces al día en una instalación tipo espina de pescado de 12 bajadas. El servicio es por inseminación artificial y se hace repaso con toro. Los terneros son criados artificialmente y las hembras permanecen durante las etapas de cría, recría y servicio en el establecimiento, hasta su ingreso al lote de vacas en producción.

Por su parte, los machos -al superar los $130 \mathrm{~kg}$ - son retirados del rodeo. El establecimiento no comparte alambrado perimetral con ningún vecino por encontrarse delimitado por dos caminos vecinales, una ruta provincial y una vía de ferrocarril en desuso.

En el predio también se halla un rebaño ovino de unas 70 cabezas para consumo propio, el cual permanece apartado del rodeo lechero, no registrando casos clínicos de enfermedad y sin movimiento de ingreso. El establecimiento registró como único ingreso externo de bovinos al rodeo, la adquisición de un toro dos años antes del brote.

Diagnóstico. Se tomaron muestras de sangre de vacas en ordeño y secas con síntomas de decaimiento, aborto reciente, diarrea, mal estado general y baja o nula producción. La sangre se extrajo con anticoagulante (EDTA) y se la procesó dentro de las 3 horas de extraída. Se realizó observación de frotis finos coloreados con Giemsa y se determinó el volumen del paquete celular a través de la técnica de microhematocrito.

Sobre el mismo tubo de microhematocrito se realizó la búsqueda de tripanosomas por observación de movilidad en la interface glóbulos blancos-plasma (buffy coat) descripta por Woo ${ }^{20}$. La tipificación del parásito como T. vivax, se realizó mediante técnica de $\mathrm{PCR}^{6}$.

Análisis económico del brote. Se trató de un estudio empírico de evaluación de los costos atribuibles al brote. En este marco, a partir de los casos clínicos diagnosticados y el tratamiento administrado, los abortos, descarte y muertes atribuibles al brote, se dedujeron los costos directos del brote en el establecimiento, siguiendo las pautas publicadas por la $\mathrm{OMS}^{3} \mathrm{e}$ investigaciones previas 9,12 .

La pauta identifica como costos directos a las pérdidas por la enfermedad en el establecimiento y las erogaciones relacionadas a los tratamientos instaurados en los animales afectados. La estimación de los costos indirectos, sugeridos en la misma pauta ${ }^{3,12}$ y relacionados a las pérdidas de mercado, interdicción y otros gastos gubernamentales, no fueron contemplados en el análisis.

Valoración del costo de las vacas descartadas, muertas y abortadas. Para ello se utilizó el modelo desarrollado por Cabrera ${ }^{2}$, disponible libremente en la red (http://dairymgt.info/tools/cow_value_resp/index. php). El modelo estima el valor de la vaca a largo plazo y lo compara con su posible reemplazo aplicando la siguiente ecuación: valor de la vaca $=$ (valor presente neto de la vaca - valor presente neto del reemplazo + costo del reemplazo - costo por venta de vaca - valor del ternero) ${ }^{2}$.

Si el valor de la vaca fue positivo, ésta debía ser retenida aún en el rodeo y en caso negativo, debía ser reemplazada. Por otro lado, si el valor fuera negativo, el reemplazo era la decisión de elección. En el marco del brote, los valores positivos de las vacas descartadas indicaron pérdidas por desecho prematuro. Para estimar el costo de los animales muertos, el valor de la vaca no incluiría el ingreso por su venta.

Estimación de la pérdida de preñez con el modelo. Para ello se compararon dos vacas de igual categoría y días en lactación, una preñada con determinados días en gestación y otra vacía. La diferencia en el valor de la vaca fue el costo de la pérdida de la preñez ${ }^{2}$. De esta manera, se cuantificaron las pérdidas por descarte, muerte y abortos durante el brote, utilizando la opción "herd analysis" del modelo para estimar el valor de la vaca.

A los efectos de todas las estimaciones, el modelo fue ajustado utilizando la información disponible en el mercado ${ }^{13}$, con datos reportados por Cattaneo ${ }^{4} \mathrm{y}$ a partir de datos colectados en el establecimiento. Las cifras incluidas en el modelo se detallan en la Tabla 1.

El otro componente de los costos directos estimados, fueron aquellos relacionados al tratamiento administrado a las vacas afectadas. El mismo, fue evaluado en aproximadamente USD 7.83 por vaca, considerando un animal de $600 \mathrm{~kg}$, una dosis de $3,5 \mathrm{mg} / \mathrm{kg}$, sumado al valor de tres días de descarte de la leche (54 1 x USD $0.31=$ USD 16.74), lo que contabiliza un costo de USD 24.57 (USD 16.74 + USD 7.83 ( $\$ 17,05$, promedio 20162017 Banco Nación Argentina) ${ }^{1,9}$.

Una vez calculados los costos de cada uno de los eventos, se procedió a la sumatoria de los mismos expresándose por vaca total y en ordeño de forma análoga ${ }^{18}$. 


\section{RESULTADOS Y DISCUSIÓN}

Desarrollo del brote. El diagnóstico de T. vivax se realizó en abril de 2017, luego de una visita al establecimiento para evaluar y muestrear animales con síntomas clínicos de enfermedad. A nueve vacas se les tomó temperatura rectal y se les extrajo sangre para realizar hematocrito y frotis. Tres de estos animales presentaron temperatura superior a $39,5^{\circ} \mathrm{C}$ y en ellas se observó el parásito en sangre, con valores de microhematocrito entre 20 y 24\%. Dada la estación del año se apreciaba abundante presencia de moscas, sin aplicarse en el establecimiento método de control alguno.

Se realizó la necropsia de una vaca que el día anterior había requerido asistencia al parto, extrayéndose un ternero a término, pero muerto. Este animal presentaba buena condición corporal, permaneciendo en decúbito luego de la asistencia, recibiendo tratamiento preventivo con solución glucosada de $\mathrm{Ca} \mathrm{y} \mathrm{Mg}$, pero murió a las pocas horas.

Los hallazgos fueron: liquido seroso en cavidad abdominal y torácica, nódulos linfáticos aumentados de tamaño, petequias sobre serosas, congestión y agrandamiento de hígado y bazo, así como congestión de meninges. Establecido el diagnóstico se procedió a tratar la totalidad de vacas adultas con diaceturato de diminazene en dosis de $3,5 \mathrm{mg} / \mathrm{kg}$ de peso por vía IM.

A partir del análisis de los registros de eventos sanitarios y reproductivos que se llevaban en el establecimiento, se estimó que el brote había comenzado entre seis y nueve semanas antes del diagnóstico etiológico, cuando se observaron los primeros abortos en vacas secas, que no presentaban la producción de leche esperada, decaían en su condición corporal y algunas de ellas morían días después.

Previamente al diagnóstico, varias de las vacas abortadas y otras que no sufrieron este evento, fueron investigadas serológicamente para determinar presencia de anticuerpos contra brucelosis, anaplasmosis, leptospirosis, IBR, DVB y neosporosis. Todos los resultados fueron negativos a brucelosis, leptospirosis y anaplasmosis.

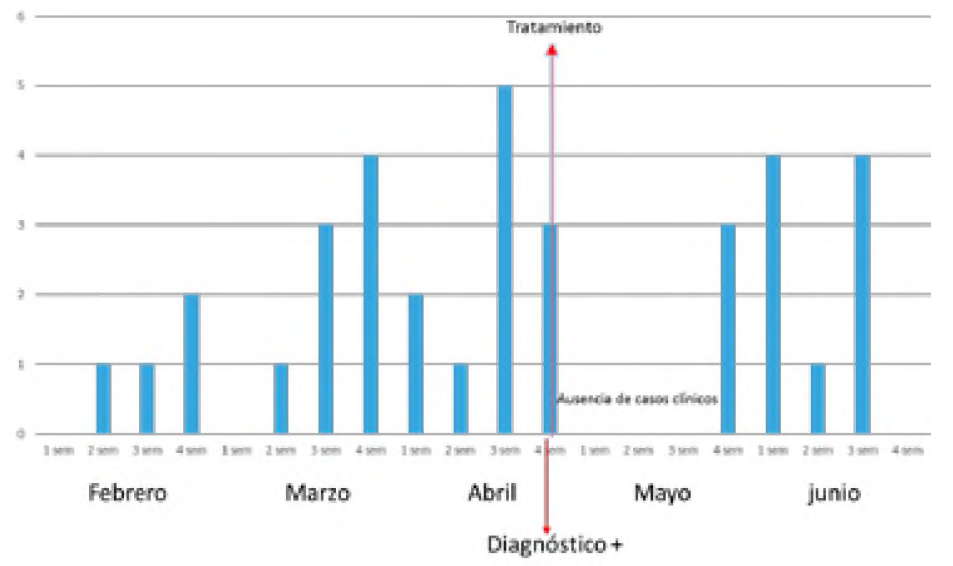

Figura 1. Detalle de presentación de casos clínicos por T. vivax, previo al diagnóstico y tratamiento y posteriores a estos.
Tabla 1. Condiciones aportadas al modelo para el cálculo de costos por descarte, muertes y abortos.

\begin{tabular}{lr}
\hline \multicolumn{1}{c}{ parámetros productivos } & 6350 \\
promedio de producción vaca / año (1) & 20,2 \\
tasa de preñez cada 21 días (\%) & 20 \\
costos reproductivos vaca / mes (USD) & 600 \\
peso promedio de vacas (Kg & \\
$\quad$ parámetros económicos & 1300 \\
costo reemplazo (USD / vaca) & 0,763 \\
valor de la vaca descartada (USD / $\mathrm{kg}$ ) & 137 \\
valor del ternero (USD) & 0,318 \\
precio de la leche (USD / kg) & 3,5 \\
grasa butirosa (\%) & 0,24 \\
costo de alimentación de la vaca en producción (USD/kg) & 0,18 \\
costo de alimentación de la vaca seca (USD/kg) & 6 \\
tasa de interés (\%)
\end{tabular}

El valor de la vaca: http://dairymgt.info/tools/cow value resp/index.php (solapa herd analysis).

Tabla 2. Costos (USD) estimados de un brote de tripanosomiasis en un rodeo lechero de Santa Fe, Argentina.

\begin{tabular}{lccccc}
\hline \multirow{2}{*}{ concepto } & \multicolumn{5}{c}{ costos estimados (USD) } \\
& mediana & promedio & mínimo & máximo & total \\
\hline muertes & 912,0 & 922,8 & 797,0 & 1168,0 & 15687,0 \\
descartes & 389,0 & 400,7 & 147,0 & 638,0 & 8013,0 \\
abortos & 270,0 & 275,0 & 349,0 & 167,0 & 29697,0 \\
tratam. ${ }^{x}$ & & & & & 5405,4 \\
total & & & & & 58802,4 \\
\hline
\end{tabular}

Nota: las estimaciones (USD) varían de acuerdo al número ordinal del parto, días en lactación y meses de preñez. ${ }^{x}$ Costos del tratamiento por el total de vacas ( $220 \times 24,57$ USD)

Las determinaciones de DVB, neosporosis e IBR, revelaron que un tercio de los individuos de ambos grupos (con y sin signología) fueron reaccionantes a las dos primeras enfermedades y casi la totalidad de los individuos muestreados (de ambos grupos) resultaron positivos a la última enfermedad.

La secuencia de aparición de animales con síntomas clínicos compatibles con la tripanosomiasis, junto a la observación del parásito (T. vivax) y la aplicación del tratamiento específico a la totalidad de vacas, se presentan en la Figura 1.

También se pudo apreciar un período ventana entre tratamiento y nueva observación de animales con sintomatología, ocurrido por efecto de la droga utilizada, que detuvo la aparición de casos clínicos, pero al superar su efecto residual volvieron a observarse animales con síntomas. Esto fue debido a la rápida metabolización y excreción del diminazene, que hace que carezca de utilidad profilácti$\mathrm{ca}^{17}$, por lo cual varios animales debieron tratarse nuevamente. 
En este marco se registraron diecisiete abortos, la mayoría ocurridos en el último tercio de la gestación. Los abortos cesaron luego del tratamiento específico, no así los decesos de vacas, que sucedieron hasta nueve semanas después de haber recibido el tratamiento específico.

La mortandad atribuida a esta parasitosis fue del $7,5 \%$ sobre el stock de vacas adultas del establecimiento al inicio del brote.

En total, 20 vacas debieron ser descartadas del tambo por haberse secado luego de sufrir la enfermedad y presentar mala condición corporal, a pesar de haber sido tratadas y contar con alimentación adecuada. Esta condición fue atribuida al deterioro en la homeostasis que produce el parásito en algunos animales ${ }^{5}$.

Ninguna otra categoría de bovinos presentes en el establecimiento, ni del rodeo de ovinos, presentó síntomas de enfermedad durante el transcurso del brote. Esto demostró la alta susceptibilidad de las vacas lecheras al T. vivax, especialmente en su etapa de pre-y post-parto.

Análisis económico. A partir de los costos del brote identificados en el marco de este estudio, se estimó una suma total de USD $58.802,4(261,4$ US\$/vaca en ordeño) por concepto de descarte, muerte, abortos y tratamientos de casos clínicos, todos estos componentes entendidos como costos directos (Tabla 1). De este total estimado, sólo un 9,20\% (USD 4.107,4 / 57.504,4) correspondió a los tratamientos aplicados a los casos, siendo las perdidas productivas, el componente esencial de los costos directos del brote.

Es necesario destacar que estas estimaciones fueron conservadoras, ya que no incluyeron aquellas pérdidas debidas a disminución de la producción entre los animales afectados en el proceso de recuperación; además de poder variar considerablemente de acuerdo a los costos de transacción, influenciados fuertemente por los precios de la vaquillona de reposición y la vaca de descarte.

Si bien existen reportes previos sobre la dinámica y brotes de tripanosomiasis bovina en Argentina ${ }^{10,15}$, no existen precedentes sobre el impacto de la enfermedad en los rodeos nacionales. La mayor parte de los datos de pérdidas en Sudamérica son estimaciones realizadas sobre ganado de carne. En la región del pantanal (Brasil) y su faja aledaña de Bolivia, las pérdidas estimadas fueron de 15 USD por animal (4\% del valor del mismo) y la relación costo/beneficio del tratamiento fue del orden de 1:10, ${ }^{19}$.

En un brote en Guyana Francesa las pérdidas fueron estimadas en $€ 33,5$ por animal ( $3 \%$ del valor en peso vivo por animal) ${ }^{8}$. En ganado lechero de Colombia, se estimó una pérdida promedio de USD 56,5 por animal ${ }^{16}$.

Dada la magnitud de la pérdida observada en el rodeo lechero local, es un punto a evaluar la utilización de drogas con efecto profiláctico como el cloruro de isometamidium en rodeos afectados y en rodeos veci- nos a estos. Esta droga previene la infección por 2-3 meses ${ }^{11}$.

Esta cobertura debería abarcar la época de mayor actividad de los insectos hematófagos (verano/otoño). La desventaja de su uso es la restricción del consumo de carne de los animales tratados durante 30 días posttratamiento. Tal inconveniente no sería tan severo para el consumo de leche, debido a la baja excreción de la droga por esta vía ${ }^{11}$.

Conclusión. La demora en el diagnóstico de la enfermedad, al ser confundida con otras patologías, posibilitó su difusión en el rodeo, magnificando las pérdidas. Esta propagación se debió posiblemente a la abundante población de vectores presentes en esta época del año, especialmente de Stomoxis calcitrans y tábanos.

La aplicación de la única droga disponible en el mercado argentino, permitió la remisión de los síntomas clínicos y la detención de la aparición de nuevos casos, pero al carecer de actividad profiláctica, algunos animales requirieron de nuevos tratamientos semanas después.

Las estimaciones económicas realizadas pueden variar de acuerdo a las características del rodeo (nivel de producción y costos de alimentación), así como las condiciones de mercado (precio de la leche, de la vaca conserva y de la vaquillona de reposición). Asimismo, el costo del tratamiento del rodeo representó una fracción menor de las pérdidas directas totales, resaltando el beneficio de un diagnóstico precoz y el tratamiento del total de los bovinos adultos del rodeo.

Agradecimientos. Al propietario del establecimiento Sr. Claudio Gorreta, al encargado del rodeo Sr. Osvaldo Garay y a los veterinarios Facundo Barra y Hernán Socin.

\section{REFERENCIAS}

1. Banco de la Nación Argentina. https://www.cotizaciondolar.com .ar/dolar-historico-BNA-2017.php.

2. Cabrera VE. 2012. A simple formulation and solution to the replacement problem: A practical tool to assess the economic cow value, the value of a new pregnancy, and the cost of a pregnancy loss. J Dairy Sci 95: 4683-4698.

3. Caspari C. 2007. Prevention and control of animal diseases worldwide: Economic analysis. Prevention versus outbreak costs. World Health Organization, 200 p.

4. Cattaneo L, Baudracco J, Lazzarini B, Ortega H. 2015. Methodology to estimate the cost of delayed pregnancy for dairy cows. An example for Argentina. R Bras Zootec 44: 226-229.

5. Coetzer JA, Tustin RC. 2005. Infectious diseases of livestock, vol. 1, 2nd edition, Oxford University Press, England, $650 \mathrm{p}$.

6. Cortez AP et al. 2009. Cathepsin L-like genes of Trypanosoma vivax from Africa and South America. Characterization, relationships and diagnostic implications. Mol Cell Probes 23: 44-51. 
7. Dagnachew S, Bezie M. 2015. Review on Trypanosoma vivax. African J of Basic \& Appl Sci 7: 41-64.

8. Desquesnes M. 2004. Livestock trypanosomoses and their vectors in Latin America. OIE-CIRAD, Paris, $190 \mathrm{p}$. On line: https://www.oie.int/doc/ged/D9818.PDF

9. Drummond MF et al. 2005. Methods for the economic evaluation of health care programmes, Third ed., Oxford Medical Publications, $379 \mathrm{p}$.

10. Filippetti CH et al. 2016. Descripción de un caso de tripanosomiasis bovina en un tambo. Rev Méd (Buenos Aires) 97: 3,46

11. Giordani F et al. 2016. The animal trypanosomiasis and their chemotherapy; a rewiew. Parasitology 143: 18621889.

12. Gethmann CP, Sauter LC, Conraths FJ. 2015. Economic analysis of animal disease outbreaks; BSE and bluetongue disease as examples. Berliner und Munchner Tierarztliche Wochennschrift 128: 478-482.

13. Mercado de Liniers S.A. http://www.mercadodeliniers. com.ar/dll/haciendal.dll/ha ciinfo000002

14. Monzón CM, Mancebo OA, Giménez JN, Russo AM. 2008. Primera descripción de T. vivax en Argentina. Vet Arg 25: 492-498.
15. Monzon CM, Mancebo OA, Giménez JN, Russo AM. 2013. Evolución de la Trypanosomosis bovina por T. vivax en Formosa (Argentina, años 2007-2012) y su potencial dispersión en el país. Rev Ibero-Latinoam Parasit 72: 3844.

16. Osorio AL et al. 2008. Trypanosoma (Duttonella) vivax: its biology, epidemiology, pathogenesis, and introduction in the New World. A review. Mem Inst Oswaldo Cruz 103: 1-13.

17. Peregrine AS, Mamman M. 1993. Pharmacology of diminazene: a review. Acta Tropica 54: 185-203.

18. Richardet MS et al. 2016. Magnitud y variación de la mastitis clínica y sus costos asociados en rodeos lecheros de Argentina. Arch Med Vet 48: 15-20.

19. Seidl A, Dávila AM, Silva R. 1999. Estimated financial impact of Trypanosoma vivax on the Brazilian Pantanal and Bolivian Lowlands. Mem Inst Oswaldo Cruz 94: 269272.

20. Woo PT. 1970. The haematocrit centrifuge technique for the diagnosis of African trypanosomiasis. Acta Trop 27 : 384-386. 